\title{
A Characterization of Jacobson Radical in $\Gamma$-Banach Algebras
}

\author{
Nilakshi Goswami \\ Department of Mathematics, Gauhati University, Guwahati, India \\ Email: nila_g2003@yahoo.co.in
}

Received August 7, 2012; revised September 25, 2012; accepted October 3, 2012

\begin{abstract}
Let $V_{1}$ and $V_{2}$ be two $\Gamma$-Banach algebras and $R_{i}$ be the right operator Banach algebra and $L_{i}$ be the left operator Banach algebra of $V_{i}(i=1,2)$. We give a characterization of the Jacobson radical for the projective tensor product $V_{1} \otimes_{\gamma} V_{2}$ in terms of the Jacobson radical for $R_{1} \otimes_{\gamma} L_{2}$. If $V_{1}$ and $V_{2}$ are isomorphic, then we show that this characterization can also be given in terms of the Jacobson radical for $R_{2} \otimes_{\gamma} L_{1}$.
\end{abstract}

Keywords: Г-Algebra; Right Quasi Regularity; Tensor Product; Operator Banach Algebra

\section{Introduction}

In $[1,2]$, using the right quasi regularity property, Kyuno and Coppage and Luh gave a characterization of Jacobson radical in $\Gamma$-rings. Many interesting results on the internal properties of Jacobson radical for $\Gamma$-rings were developed in [2-5] by different research workers. In [6], some of these results are extended to $\Gamma$-algebras. In this paper, we consider two $\Gamma$-Banach algebras $V_{1}$ and $V_{2}$ and consider their projective tensor product $V_{1} \otimes_{\gamma} V_{2}$. Let $R_{i}$ be the right operator Banach algebra and $L_{i}$ be the left operator Banach algebra of $V_{i}(i=1,2)$. We give a characterization of Jacobson radical $J\left(V_{1} \otimes_{\gamma} V_{2}\right)$ in terms of $J\left(R_{1} \otimes_{\gamma} L_{2}\right)$

Before going to present our main results, we first give some basic terminologies (refer to [5-12]) which are needed in our discussion.

\section{Definition 1.1}

Let $X$ be a ring having the unit element $e$. A new multiplication called the circle composition (refer to [5]) on $X$ is defined by: $x \cdot x^{\prime}=x+x^{\prime}-x x^{\prime}$. This composition makes sense even when $X$ does not have the unit element. An element $x$ of $X$ is said to be right quasi regular if it has a right quasi inverse w.r.t. this composition, i.e., there exists $x^{\prime} \in X$ such that $x \cdot x^{\prime}=x+x^{\prime}-x x^{\prime}=0$.

\section{Definition 1.2}

Let $V$ and $\Gamma$ be two linear spaces over a field $F$. $V$ is said to be a $\Gamma$-algebra over $F$ if, for $x, y, z \in V ; \alpha$, $\beta \in \Gamma ; a \in F$, the following conditions are satisfied:

1) $x \alpha y \in V$;

2) $(x \alpha y) \beta z=x \alpha(y \beta z)$;
3) $a(x \alpha y)=(a x) \alpha y=x(a \alpha) y=x \alpha(a y)$;

4) $x \alpha(y+z)=x \alpha y+x \alpha z$, $x(\alpha+\beta) y=x \alpha y+x \beta y$, $(x+y) \alpha z=x \alpha z+y \alpha z$.

The $\Gamma$-algebra is denoted by $(V, \Gamma)$. If $V$ and $\Gamma$ are normed linear spaces over $F$, then $\Gamma$-algebra $(V, \Gamma)$ is called a $\Gamma$-normed algebra if conditions 1 ) to 4 ) hold and further

5) $\|x \alpha y\| \leq\|x\| \cdot\|\alpha\| \cdot\|y\|$ holds.

A $\Gamma$-normed algebra $(V, \Gamma)$ is called a $\Gamma$-Banach algebra if $V$ is a Banach space. Any Banach algebra can be regarded as a $\Gamma$-Banach algebra by suitably choosing $\Gamma$.

\section{Definition 1.3}

A subset $I$ of a $\Gamma$-Banach algebra $V$ is said to be a right (left) $\Gamma$-ideal of $V$ if

1) $I$ is a subspace of $V$ (in the vector space sense);

2) $x \alpha y \in I(y \alpha x \in I) \forall x \in I, \alpha \in \Gamma ; y \in V$ i.e., $I \Gamma V \subseteq I(V \Gamma I \subseteq I)$.

A right $\Gamma$-ideal, which is a left $\Gamma$-ideal as well, is called a two-sided $\Gamma$-ideal or simply a $\Gamma$-ideal.

Definition 1.4

Let $V$ be a $\Gamma$-Banach algebra and let $x \in V, \quad \alpha \in \Gamma$. Then the mapping $[\alpha, x]$ defined by $y[\alpha, x]=y \alpha x \forall y \in V$ is a right Banach space endomorphism of $V$. The collection $R$ of all endomorphisms generated by $[\alpha, x] ; \alpha \in \Gamma, x \in V$, is a Banach algebra under the operations:

$$
\begin{aligned}
& {[\alpha, x]+[\alpha, y]=[\alpha, x+y],} \\
& {[\alpha, x]+[\beta, x]=[\alpha+\beta, x]}
\end{aligned}
$$


where $a \in F$,

$$
a[\alpha, x]=[\alpha, a x]=[a \alpha, x]
$$

$$
[\alpha, x][\beta, y]=[\alpha, x \beta y], \alpha, \beta \in \Gamma,
$$

and the norm:

$$
\|[\alpha, x]\|=\|\alpha\|_{\Gamma} \cdot\|x\|_{V} .
$$

This Banach algebra is termed as the right operator Banach algebra of $\Gamma$-Banach algebra $V$. We can similarly define the left operator Banach algebra $L$ of $V$ as the Banach algebra generated by the set of all left endomorphisms of $V$ in the form $[x, \alpha]$ where

$$
[x, \alpha] y=x \alpha y \forall y \in V .
$$

\section{Definition 1.5}

Let $V$ and $V^{\prime}$ be $\Gamma$-Banach algebras over $F$ and $\phi$ : $V \rightarrow V^{\prime}$ be a mapping. Then $\phi$ is called a $\Gamma$-Banach algebra homomorphism if

1) $\phi(a x+b y)=a \phi(x)+b \phi(y)$ and

2) $\phi(x \alpha y)=\phi(x) \alpha \phi(y)$ for all $x, y \in V ; \alpha \in \Gamma$ and $a, b \in F$

\section{Definition 1.6}

Let $X$ and $Y$ be two normed spaces. The projective tensor norm $\|\cdot\|_{\gamma}$ on $X \otimes Y$ is defined as:

$$
\|u\|_{\gamma}=\inf \left\{\sum_{i}\left\|x_{i}\right\| \cdot\left\|y_{i}\right\|: u=\sum_{i} x_{i} \otimes y_{i}\right\}
$$

where the infimum is taken over all (finite) representations of $u$. The completion of $\left(X \otimes Y,\|.\|_{\gamma}\right)$ is called the projective tensor product of $X$ and $Y$, and is denoted by $X \otimes_{\gamma} Y$.

Let $(V, \Gamma)$ and $\left(V^{\prime}, \Gamma^{\prime}\right)$ be $\Gamma$-Banach algebras over $F_{1}$ and $F_{2}$ isomorphic to $F$. The projective tensor product $(V, \Gamma) \otimes_{\gamma}\left(V^{\prime}, \Gamma^{\prime}\right)$ with the projective tensor norm is a $\Gamma \otimes \Gamma^{\prime}$-Banach algebra over $F$, where a multiplication is defined by the formula:

$$
(x \otimes y)(\alpha \otimes \beta)\left(x^{\prime} \otimes y^{\prime}\right)=\left(x \alpha x^{\prime}\right) \otimes\left(y^{\prime} \beta y\right) .
$$

where $x, y \in V ; x^{\prime}, y^{\prime} \in V^{\prime} ; \alpha \in \Gamma, \beta \in \Gamma^{\prime}$.

\section{Definition 1.7}

Let $V$ be a $\Gamma$-Banach algebra. Let $\alpha \in \Gamma$. An element $x$ in $V$ is said to be $\alpha$-right quasi regular with $\alpha$-right quasi inverse $y$ if $x+y-x \alpha y=0 . x$ is said to be a right quasi regular element of $V$ if it is $\alpha$-right quasi regular for each $\alpha \in \Gamma$.

Equivalently, an element $x \in V$ is called right quasi regular if for any $\alpha \in \Gamma$, there exist $\gamma_{i} \in \Gamma, v_{i} \in V$, $i=1,2, \cdots, n$ such that

$$
v \alpha x+\sum_{i=1}^{n} v \gamma_{i} v_{i}-\sum_{i=1}^{n} v \alpha x \gamma_{i} v_{i}=0 \forall v \in V
$$

An ideal $I$ of $V$ is said to be right quasi regular if each of its elements is right quasi regular.

We have, right quasi regularity is a radical property in an algebra. The maximal right quasi regular ideal is called the Jacobson radical of $V$ and it is denoted by $J(V)$.

\section{Main Results}

In [6], we have the following Lemma regarding right quasi regularity of a $\Gamma$-Banach algebra and its operator algebra.

Lemma 2.1

An element $x$ of a $\Gamma$-Banach algebra $V$ is right quasi regular if and only if for all $\alpha \in \Gamma,[\alpha, x]$ is right quasi regular in the right operator Banach algebra $R$ of $V$.

Extending this result to the projective tensor product of $\Gamma$-Banach algebras, we prove,

\section{Lemma 2.2}

Let $V$ and $V^{\prime}$ be two $\Gamma$ and $\Gamma^{\prime}$-Banach algebras respectively. Let $R$ be the right operator Banach algebra of $V$ and $L$ be the left operator Banach algebra of $V^{\prime}$. If $\sum_{i} x_{i} \otimes x_{i}^{\prime}$ is right quasi regular in $V \otimes_{\gamma} V^{\prime}$, then $\sum_{i}\left[\alpha, x_{i}\right] \otimes\left[x_{i}^{\prime}, \alpha^{\prime}\right]$ is right quasi regular in $R \otimes_{\gamma} L$ for $\alpha \otimes \alpha^{\prime} \in \Gamma \otimes \Gamma^{\prime}$, and conversely.

Proof. Since $\sum_{i} x_{i} \otimes x_{i}^{\prime}$ is right quasi regular in $V \otimes_{\gamma} V^{\prime}$, so, for any $\alpha \otimes \alpha^{\prime} \in \Gamma \otimes \Gamma^{\prime}$, there exist $\eta_{j}=\sum_{n} \gamma_{j n} \otimes \gamma_{j n}^{\prime} \in \Gamma \otimes \Gamma^{\prime}, \quad p_{j}=\sum_{m} x_{j m} \otimes x_{j m}^{\prime} \in V \otimes_{\gamma} V^{\prime}$, $j=1,2, \cdots, n_{0}$ such that for any $q=\sum_{k} v_{k} \otimes v_{k}^{\prime} \in V \otimes_{\gamma} V^{\prime}$,

$$
\begin{aligned}
& q\left(\alpha \otimes \alpha^{\prime}\right)\left(\sum_{i} x_{i} \otimes x_{i}^{\prime}\right)+\sum_{j=1}^{n_{0}} q \eta_{j} p_{j}-\sum_{j=1}^{n_{0}} q\left(\alpha \otimes \alpha^{\prime}\right)\left(\sum_{i} x_{i} \otimes x_{i}^{\prime}\right) \eta_{j} p_{j}=0 \\
& \Rightarrow\left(\sum_{k} v_{k} \otimes v_{k}^{\prime}\right)\left(\alpha \otimes \alpha^{\prime}\right)\left(\sum_{i} x_{i} \otimes x_{i}^{\prime}\right)+\sum_{j=1}^{n_{0}}\left(\sum_{k} v_{k} \otimes v_{k}^{\prime}\right)\left(\sum_{n} \gamma_{j n} \otimes \gamma_{j n}^{\prime}\right)\left(\sum_{m} x_{j m} \otimes x_{j m}^{\prime}\right) \\
& \quad-\sum_{j=1}^{n_{0}}\left(\sum_{k} v_{k} \otimes v_{k}^{\prime}\right)\left(\alpha \otimes \alpha^{\prime}\right)\left(\sum_{i} x_{i} \otimes x_{i}^{\prime}\right)\left(\sum_{n} \gamma_{j n} \otimes \gamma_{j n}^{\prime}\right)\left(\sum_{m} x_{j m} \otimes x_{j m}^{\prime}\right)=0 \\
& \Rightarrow \sum_{k, i} v_{k} \alpha x_{i} \otimes x_{i}^{\prime} \alpha^{\prime} v_{k}^{\prime}+\sum_{j=1}^{n_{0}}\left(\sum_{k, n, m} v_{k} \gamma_{j n} x_{j m} \otimes x_{j m}^{\prime} \gamma_{j n}^{\prime} v_{k}^{\prime}\right)-\sum_{j=1}^{n_{0}}\left(\sum_{k, i, n, m} v_{k} \alpha x_{i} \gamma_{j n} x_{j m} \otimes x_{j m}^{\prime} \gamma_{j n}^{\prime} x_{i}^{\prime} \alpha^{\prime} v_{k}^{\prime}\right)=0
\end{aligned}
$$


Let $x=\sum_{i}\left[\alpha, x_{i}\right] \otimes\left[x_{i}^{\prime}, \alpha^{\prime}\right]$. We take $y=\sum_{j=1}^{n_{0}} \sum_{n, m}\left[\gamma_{j n}, x_{j m}\right] \otimes\left[x_{j m}^{\prime}, \gamma_{j n}^{\prime}\right]$

Now,

$$
\begin{aligned}
(x+y-x y)\left(\sum_{k} v_{k} \otimes v_{k}^{\prime}\right)= & \left(\left(\sum_{i}\left[\alpha, x_{i}\right] \otimes\left[x_{i}^{\prime}, \alpha^{\prime}\right]\right)+\sum_{j=1}^{n_{0}} \sum_{n, m}\left[\gamma_{j n}, x_{j m}\right] \otimes\left[x_{j m}^{\prime}, \gamma_{j n}^{\prime}\right]\right. \\
& \left.-\left(\sum_{i}\left[\alpha, x_{i}\right] \otimes\left[x_{i}^{\prime}, \alpha^{\prime}\right]\right)\left(\sum_{j=1}^{n_{0}} \sum_{n, m}\left[\gamma_{j n}, x_{j m}\right] \otimes\left[x_{j m}^{\prime}, \gamma_{j n}^{\prime}\right]\right)\right)\left(\sum_{k} v_{k} \otimes v_{k}^{\prime}\right) \\
= & \left(\sum_{i}\left[\alpha, x_{i}\right] \otimes\left[x_{i}^{\prime}, \alpha^{\prime}\right]\right)\left(\sum_{k} v_{k} \otimes v_{k}^{\prime}\right)+\sum_{j=1}^{n_{0}} \sum_{n, m}\left[\gamma_{j n}, x_{j m}\right] \otimes\left[x_{j m}^{\prime}, \gamma_{j n}^{\prime}\right]\left(\sum_{k} v_{k} \otimes v_{k}^{\prime}\right) \\
& -\left(\sum_{i}\left[\alpha, x_{i}\right] \otimes\left[x_{i}^{\prime}, \alpha^{\prime}\right]\right)\left(\sum_{j=1}^{n_{0}} \sum_{n, m}\left[\gamma_{j n}, x_{j m}\right] \otimes\left[x_{j m}^{\prime}, \gamma_{j n}^{\prime}\right]\right)\left(\sum_{k} v_{k} \otimes v_{k}^{\prime}\right) \\
= & \sum_{k, i} v_{k} \alpha x_{i} \otimes x_{i}^{\prime} \alpha^{\prime} v_{k}^{\prime}+\sum_{j=1}^{n_{0}}\left(\sum_{k, n, m} v_{k} \gamma_{j n} x_{j m} \otimes x_{j m}^{\prime} \gamma_{j n}^{\prime} v_{k}^{\prime}\right)-\sum_{j=1}^{n_{0}}\left(\sum_{k, i, n, m} v_{k} \alpha x_{i} \gamma_{j n} x_{j m} \otimes x_{j m}^{\prime} \gamma_{j n}^{\prime} x_{i}^{\prime} \alpha^{\prime} v_{k}^{\prime}\right)=0
\end{aligned}
$$

(by (2.1)).

But, $\sum_{k} v_{k} \otimes v_{k}^{\prime} \in V \otimes{ }_{\gamma} V^{\prime}$ is arbitrary.

So, $x+y-x y=0$. Thus, $x$, i.e., $\sum_{i}\left[\alpha, x_{i}\right] \otimes\left[x_{i}^{\prime}, \alpha^{\prime}\right]$ is right quasi regular in $R \otimes_{\gamma} L$.

The converse follows in the same way.

In [13], we have defined the following ideal for the projective tensor product of $V$ and $V^{\prime}$.

\section{Lemma 2.3}

Let $V$ and $V^{\prime}$ be two $\Gamma$ and $\Gamma^{\prime}$-Banach algebras respectively. Let $R$ be the right operator Banach algebra of $V$ and $L$ be the left operator Banach algebra of $V^{\prime}$. Let $J$ be an ideal of $R \otimes_{\gamma} L$. We define:

$$
J^{0}=\left\{\left(\sum_{i} x_{i} \otimes x_{i}^{\prime}\right) \in V \otimes_{\gamma} V^{\prime}: \sum_{i}\left[\Gamma, x_{i}\right] \otimes\left[x_{i}^{\prime}, \Gamma^{\prime}\right] \subseteq J\right\}
$$

where $\left[\Gamma, x_{i}\right]=\left\{\sum_{j}\left[\alpha_{j}, x_{i}\right]: \alpha_{j} \in \Gamma\right\}$, and

$$
\left[x_{i}^{\prime}, \Gamma^{\prime}\right]=\left\{\sum_{j}\left[x_{i}^{\prime}, \alpha_{j}^{\prime}\right]: \alpha_{j}^{\prime} \in \Gamma^{\prime}\right\}
$$

Then $J^{0}$ is an ideal of $V \otimes_{\gamma} V^{\prime}$.

Using the above defined ideal, now, we give the characterization of Jacobson radical for the projective tensor product of two $\Gamma$-Banach algebras $V_{i}(i=1,2)$ in terms of the Jacobson radical of the projective tensor product of corresponding right and left operator Banach algebras.

\section{Theorem 2.4}

Let $V_{i}$ be a $\Gamma$-Banach algebra (over $F$ ) with right operator Banach algebra $R_{i}$ and left operator Banach algebra $L_{i}(i=1,2)$ respectively. Then the Jacobson radical of $V_{1} \otimes_{\gamma} V_{2}$ is given by: $J\left(V_{1} \otimes_{\gamma} V_{2}\right)=\left[J\left(R_{1} \otimes_{\gamma} L_{2}\right)\right]^{0}$.
Proof. Let $\sum_{i} x_{i} \otimes x_{i}^{\prime} \in J\left(V_{1} \otimes_{\gamma} V_{2}\right)$.

Then $\sum_{i} x_{i} \otimes x_{i}^{\prime}$ is a right quasi regular element of $V_{1} \otimes_{\gamma} V_{2}$. By Lemma 2.2, for any $\alpha, \alpha^{\prime} \in \Gamma$, $\sum_{i}\left[\alpha, x_{i}\right] \otimes\left[x_{i}^{\prime}, \alpha^{\prime}\right]$ is a right quasi regular element of $R_{1} \otimes_{\gamma} L_{2}$, i.e.,

$$
\sum_{i}\left[\alpha, x_{i}\right] \otimes\left[x_{i}^{\prime}, \alpha^{\prime}\right] \in J\left(R_{1} \otimes_{\gamma} L_{2}\right) .
$$

So,

$$
\sum_{i}\left[\Gamma, x_{i}\right] \otimes\left[x_{i}^{\prime}, \Gamma\right] \subseteq J\left(R_{1} \otimes_{\gamma} L_{2}\right) .
$$

Hence,

$$
\sum_{i} x_{i} \otimes x_{i}^{\prime} \in\left[J\left(R_{1} \otimes_{\gamma} L_{2}\right)\right]^{0} .
$$

Thus,

$$
J\left(V_{1} \otimes_{\gamma} V_{2}\right) \subseteq\left[J\left(R_{1} \otimes_{\gamma} L_{2}\right)\right]^{0} .
$$

Conversely, let

$$
\sum_{i} x_{i} \otimes x_{i}^{\prime} \in\left[J\left(R_{1} \otimes_{\gamma} L_{2}\right)\right]^{0} .
$$

Then

$$
\sum_{i}\left[\Gamma, x_{i}\right] \otimes\left[x_{i}^{\prime}, \Gamma\right] \subseteq J\left(R_{1} \otimes_{\gamma} L_{2}\right) .
$$

So, for any $\alpha, \alpha^{\prime} \in \Gamma, \sum_{i}\left[\alpha, x_{i}\right] \otimes\left[x_{i}^{\prime}, \alpha^{\prime}\right]$ is a right quasi regular element of $R_{1} \otimes_{\gamma} L_{2}$. By Lemma 2.2, $\sum_{i} x_{i} \otimes x_{i}^{\prime}$ is a right quasi regular element of $V_{1} \otimes_{\gamma} V_{2}$, i.e. $\sum_{i} x_{i} \otimes x_{i}^{\prime} \in J\left(V_{1} \otimes_{\gamma} V_{2}\right)$ So, 
$\left[J\left(R_{1} \otimes_{\gamma} L_{2}\right)\right]^{0} \subseteq J\left(V_{1} \otimes_{\gamma} V_{2}\right)$.

Thus, $J\left(V_{1} \otimes_{\gamma} V_{2}\right)=\left[J\left(R_{1} \otimes_{\gamma} L_{2}\right)\right]^{0}$.

Let the $\Gamma$-Banach algebras $V_{1}$ and $V_{2}$ are isomorphic. In that case, we have the following result.

\section{Theorem 2.5}

Let $V_{i}$ be a $\Gamma$-Banach algebra (over $F$ ) with right operator Banach algebra $R_{i}$ and left operator Banach algebra $L_{i}(i=1,2)$ respectively. If there exists a $\Gamma$-Banach algebra isomorphism $f$ from $V_{1}$ onto $V_{2}$, then $R_{1} \otimes_{\gamma} L_{2}$ is a homomorphic image of $R_{2} \otimes_{\gamma} L_{1}$.

Proof. Let $\sum_{n} r_{n} \otimes l_{n} \in R_{2} \otimes_{\gamma} L_{1}$, where $l_{n}=\left[y_{n}, \beta_{n}\right]$, $r_{n}=\left[\alpha_{n}^{\prime}, x_{n}^{\prime}\right]$. We define $\phi: R_{2} \otimes_{\gamma} L_{1} \rightarrow R_{1} \otimes_{\gamma} L_{2}$ by

$$
\begin{aligned}
\phi\left(\sum_{n} r_{n} \otimes l_{n}\right) & =\phi\left(\sum_{n}\left[\alpha_{n}^{\prime}, x_{n}^{\prime}\right] \otimes\left[y_{n}, \beta_{n}\right]\right) \\
& =\sum_{n}\left[\alpha_{n}^{\prime}, x_{n}\right] \otimes\left[f\left(y_{n}\right), \beta_{n}\right],
\end{aligned}
$$

where $x_{n}^{\prime}=f\left(x_{n}\right), \quad x_{n} \in V_{1}$.

Let $r_{1}^{*} \in R_{1}^{*}$ (The dual space of $R_{1}$ ).

We define $r_{2}^{*}: R_{2} \rightarrow C$ by $r_{2}^{*}\left(\left[\alpha^{\prime}, x^{\prime}\right]\right)=r_{1}^{*}\left(\left[\alpha^{\prime}, x\right]\right)$, where $x^{\prime}=f(x)$.

Then $r_{2}^{*} \in R_{2}^{*}$.

Similarly, for $l_{2}^{*} \in L_{2}^{*}$, we can define $l_{1}^{*} \in L_{1}^{*}$ by $l_{1}^{*}([y, \beta])=l_{2}^{*}([f(y), \beta])$.

Now, let

$$
\sum_{n} r_{n} \otimes l_{n}=\sum_{m} \tilde{r}_{m} \otimes \tilde{l}_{m}
$$

where

$$
\begin{aligned}
& \tilde{r}_{m}=\left[\tilde{\alpha}_{m}^{\prime}, \tilde{x}_{m}^{\prime}\right], \tilde{l}_{m}=\left[\tilde{y}_{m}, \tilde{\beta}_{m}\right] \\
& \Rightarrow\left(\sum_{n} r_{n} \otimes l_{n}\right)(h, k)=\left(\sum_{m} \tilde{r}_{m} \otimes \tilde{l}_{m}\right)(h, k) \\
& \forall h \in R_{2}^{*}, k \in L_{1}^{*} .
\end{aligned}
$$

In particular, taking $h=r_{2}^{*}, \quad k=l_{1}^{*}$, we get,

$$
\begin{aligned}
& \left(\sum_{n} r_{n} \otimes l_{n}\right)\left(r_{2}^{*}, l_{1}^{*}\right)=\left(\sum_{m} \tilde{r}_{m} \otimes \tilde{l}_{m}\right)\left(r_{2}^{*}, l_{1}^{*}\right) \\
& \Rightarrow \sum_{n} r_{2}^{*}\left(r_{n}\right) l_{1}^{*}\left(l_{n}\right)=\sum_{m} r_{2}^{*}\left(\tilde{r}_{m}\right) \otimes l_{1}^{*}\left(\tilde{l}_{m}\right) \\
& \Rightarrow \sum_{n} r_{2}^{*}\left(\left[\alpha_{n}^{\prime}, x_{n}^{\prime}\right]\right) l_{1}^{*}\left(\left[y_{n}, \beta_{n}\right]\right) \\
& =\sum_{m} r_{2}^{*}\left(\left[\tilde{\alpha}_{m}^{\prime}, \tilde{x}_{m}^{\prime}\right]\right) l_{1}^{*}\left(\left[\tilde{y}_{m}, \tilde{\beta}_{m}\right]\right) \\
& \Rightarrow \sum_{n} r_{1}^{*}\left(\left[\alpha_{n}^{\prime}, x_{n}\right]\right) l_{2}^{*}\left(\left[f\left(y_{n}\right), \beta_{n}\right]\right) \\
& =\sum_{m} r_{1}^{*}\left(\left[\tilde{\alpha}_{m}^{\prime}, \tilde{x}_{m}\right]\right) l_{2}^{*}\left(\left[f\left(\tilde{y}_{m}\right), \tilde{\beta}_{m}\right]\right),
\end{aligned}
$$

where $x_{n}^{\prime}=f\left(x_{n}\right)$, and $\tilde{x}_{m}^{\prime}=f\left(\tilde{x}_{m}\right)$.

$$
\begin{aligned}
& \Rightarrow\left(\sum_{n}\left[\alpha_{n}^{\prime}, x_{n}\right] \otimes\left[f\left(y_{n}\right), \beta_{n}\right]\right)\left(r_{1}^{*}, l_{2}^{*}\right) \\
& =\left(\sum_{m}\left[\tilde{\alpha}_{m}^{\prime}, \tilde{x}_{m}\right] \otimes\left[f\left(\tilde{y}_{m}\right), \tilde{\beta}_{m}\right]\right)\left(r_{1}^{*}, l_{2}^{*}\right) \\
& \Rightarrow\left(\phi\left(\sum_{n} r_{n} \otimes l_{n}\right)\right)\left(r_{1}^{*}, l_{2}^{*}\right)=\left(\phi\left(\sum_{m} \tilde{r}_{m} \otimes \tilde{l}_{m}\right)\right)\left(r_{1}^{*}, l_{2}^{*}\right) .
\end{aligned}
$$

But $r_{1}^{*} \in R_{1}^{*}$ and $l_{2}^{*} \in L_{2}^{*}$ are arbitrary. So,

$\phi\left(\sum_{n} r_{n} \otimes l_{n}\right)=\phi\left(\sum_{m} \tilde{r}_{m} \otimes \tilde{l}_{m}\right)$ Thus $\phi$ is well defined.

Now, Let $a, b \in F$. Then

$$
\begin{aligned}
& \phi\left(a \sum_{n} r_{n} \otimes l_{n}+b \sum_{m} \tilde{r}_{m} \otimes \tilde{l}_{m}\right)=\phi\left(\sum_{n} a r_{n} \otimes l_{n}+\sum_{m} b \tilde{r}_{m} \otimes \tilde{l}_{m}\right) \\
& =\phi\left(\sum_{n} a\left[\alpha_{n}^{\prime}, x_{n}^{\prime}\right] \otimes\left[y_{n}, \beta_{n}\right]+\sum_{m} b\left[\tilde{\alpha}_{m}^{\prime}, \tilde{x}_{m}^{\prime}\right] \otimes\left[\tilde{y}_{m}, \tilde{\beta}_{m}\right]\right) \\
& =\phi\left(\sum_{n}\left[a \alpha_{n}^{\prime}, x_{n}^{\prime}\right] \otimes\left[y_{n}, \beta_{n}\right]+\sum_{m}\left[b \tilde{\alpha}_{m}^{\prime}, \tilde{x}_{m}^{\prime}\right] \otimes\left[\tilde{y}_{m}, \tilde{\beta}_{m}\right]\right) \\
& =\sum_{n}\left[a \alpha_{n}^{\prime}, x_{n}\right] \otimes\left[f\left(y_{n}\right), \beta_{n}\right] \\
& +\sum_{m}\left[b \tilde{\alpha}_{m}^{\prime}, \tilde{x}_{m}\right] \otimes\left[f\left(\tilde{y}_{m}\right), \tilde{\beta}_{m}\right],
\end{aligned}
$$

where $x_{n}^{\prime}=f\left(x_{n}\right)$, and $\tilde{x}_{m}^{\prime}=f\left(\tilde{x}_{m}\right)$.

$$
\begin{aligned}
= & \sum_{n} a\left[\alpha_{n}^{\prime}, x_{n}\right] \otimes\left[f\left(y_{n}\right), \beta_{n}\right] \\
& +\sum_{m} b\left[\tilde{\alpha}_{m}^{\prime}, \tilde{x}_{m}\right] \otimes\left[f\left(\tilde{y}_{m}\right), \tilde{\beta}_{m}\right] \\
= & a\left(\sum_{n}\left[\alpha_{n}^{\prime}, f\left(x_{n}\right)\right] \otimes\left[f\left(y_{n}\right), \beta_{n}\right]\right) \\
& +b\left(\sum_{m}\left[\alpha_{m}^{\prime}, f\left(x_{m}^{\prime}\right)\right] \otimes\left[f\left(y_{m}^{\prime}\right), \beta_{m}^{\prime}\right]\right) \\
= & a \phi\left(\sum_{n} r_{n} \otimes l_{n}\right)+b \phi\left(\sum_{m} \tilde{r}_{m} \otimes \tilde{l}_{m}\right)
\end{aligned}
$$

Again,

$$
\begin{aligned}
& \phi\left(\left(\sum_{n} r_{n} \otimes l_{n}\right)\left(\sum_{m} \tilde{r}_{m} \otimes \tilde{I}_{m}\right)\right)=\phi\left(\sum_{n, m} r_{n} \tilde{r}_{m} \otimes \tilde{I}_{m} l_{n}\right) \\
& =\phi\left(\sum_{n, m}\left[\alpha_{n}^{\prime}, x_{n}^{\prime}\right]\left[\tilde{\alpha}_{m}^{\prime}, \tilde{x}_{m}^{\prime}\right] \otimes\left[\tilde{y}_{m}, \tilde{\beta}_{m}\right]\left[y_{n}, \beta_{n}\right]\right) \\
& =\phi\left(\sum_{n, m}\left[\alpha_{n}^{\prime}, x_{n}^{\prime} \tilde{\alpha}_{m}^{\prime} \tilde{x}_{m}^{\prime}\right] \otimes\left[\tilde{y}_{m} \tilde{\beta}_{m} y_{n}, \beta_{n}\right]\right)
\end{aligned}
$$

We have, $x_{n}^{\prime}, \tilde{x}_{m}^{\prime} \in V_{2}$. So, there exist $x_{n}, \tilde{x}_{m} \in V_{1}$ such that $x_{n}^{\prime}=f\left(x_{n}\right), \tilde{x}_{m}^{\prime}=f\left(\tilde{x}_{m}\right)$.

Now, $x_{n} \tilde{\alpha}_{m}^{\prime} \tilde{x}_{m} \in V_{1}$ and

$$
f\left(x_{n} \tilde{\alpha}_{m}^{\prime} \tilde{x}_{m}\right)=f\left(x_{n}\right) \tilde{\alpha}_{m}^{\prime} f\left(\tilde{x}_{m}\right)=x_{n}^{\prime} \tilde{\alpha}_{m}^{\prime} \tilde{x}_{m}^{\prime}
$$

So, the expression (2.2) is equal to 


$$
\begin{aligned}
& \sum_{n, m}\left[\alpha_{n}^{\prime}, x_{n} \tilde{\alpha}_{m}^{\prime} \tilde{x}_{m}\right] \otimes\left[f\left(\tilde{y}_{m} \tilde{\beta}_{m} y_{n}\right), \beta_{n}\right] \\
= & \sum_{n, m}\left[\alpha_{n}^{\prime}, x_{n} \tilde{\alpha}_{m}^{\prime} \tilde{x}_{m}\right] \otimes\left[f\left(\tilde{y}_{m}\right) \tilde{\beta}_{m} f\left(y_{n}\right), \beta_{n}\right] \\
= & \sum_{n, m}\left[\alpha_{n}^{\prime}, x_{n}\right]\left[\tilde{\alpha}_{m}^{\prime}, \tilde{x}_{m}\right] \otimes\left[f\left(\tilde{y}_{m}\right), \tilde{\beta}_{m}\right]\left[f\left(y_{n}\right), \beta_{n}\right] \\
= & \left(\sum_{n}\left[\alpha_{n}^{\prime}, x_{n}\right] \otimes\left[f\left(y_{n}\right), \beta_{n}\right]\right) \\
& \cdot\left(\sum_{m}\left[\tilde{\alpha}_{m}^{\prime}, \tilde{x}_{m}\right] \otimes\left[f\left(\tilde{y}_{m}\right), \tilde{\beta}_{m}\right]\right) \\
= & \phi\left(\sum_{n} r_{n} \otimes l_{n}\right) \phi\left(\sum_{m} \tilde{r}_{m} \otimes \tilde{l}_{m}\right)
\end{aligned}
$$

So, $\phi: R_{2} \otimes_{\gamma} L_{1} \rightarrow R_{1} \otimes_{\gamma} L_{2} \quad$ is a homomorphism.

Since $f$ is onto, so, $\phi$ is also onto. Also, it can be shown that $\phi$ is one-one.

Thus, $R_{1} \otimes_{\gamma} L_{2}=\phi\left(R_{2} \otimes_{\gamma} L_{1}\right)$.

\section{Corollary 2.6}

Let the $\Gamma$-Banach algebras $V_{1}$ and $V_{2}$, as defined in Theorem 2.4 are isomorphic. Then we have,

$$
J\left(V_{1} \otimes_{\gamma} V_{2}\right)=\left[J\left(\phi\left(R_{2} \otimes_{\gamma} L_{1}\right)\right)\right]^{0}
$$

\section{Remark 2.7}

If the isomorphism $f$ from $V_{1}$ onto $V_{2}$ is isometric, then we can show that $\phi: R_{2} \otimes_{\gamma} L_{1} \rightarrow R_{1} \otimes_{\gamma} L_{2}$ is also an isometry. So, in that case,

$$
J\left(V_{1} \otimes_{\gamma} V_{2}\right) \cong\left[J\left(\phi\left(R_{2} \otimes_{\gamma} L_{1}\right)\right)\right]^{0} .
$$

The notion of direct summand for $\Gamma$-rings is discussed in [10] by Booth. For a $\Gamma$-Banach algebra $V$, an ideal $P$ is called direct summand if there exists a $\Gamma$-ideal $Q$ of $V$ such that every element $v$ of $V$ is uniquely expressible in the form $v=p+q, \quad p \in P, q \in Q$, and $V$ is written as $V=P \oplus Q$. Clearly, if $V=P \oplus Q$, then for $p \in P$, $q \in Q, \quad p \alpha q=0 \forall \alpha \in \Gamma$.

Now, we prove:

\section{Deduction 2.8}

If $P$ is the direct summand for the $\Gamma$-Banach algebra $V_{1} \otimes_{\gamma} V_{2}$, then $J(P)$ is the direct summand for $J\left(V_{1} \otimes_{\gamma} V_{2}\right)$.

Proof. Let $V_{1} \otimes_{\gamma} V_{2}=P \oplus Q$ Clearly, $J(P) \cap J(Q)=\{0\}$.

Let $x \in J\left(V_{1} \otimes_{\gamma} V_{2}\right)$ and $x=p+q$, where $p \in P$, $q \in Q$.

Since $x$ is right quasi regular in $V_{1} \otimes_{\gamma} V_{2}$, so, for any $\alpha \otimes \alpha^{\prime} \in \Gamma \otimes \Gamma$, we have, there exists $y \in V_{1} \otimes_{\gamma} V_{2}$ such that $x+y-x\left(\alpha \otimes \alpha^{\prime}\right) y=0$.

Let $y=p_{1}+q_{1}$, where $p_{1} \in P, q_{1} \in Q$.

So,

$$
\begin{aligned}
& (p+q)+\left(p_{1}+q_{1}\right)-(p+q)\left(\alpha \otimes \alpha^{\prime}\right)\left(p_{1}+q_{1}\right)=0 \\
& \Rightarrow\left(p+p_{1}-p\left(\alpha \otimes \alpha^{\prime}\right) p_{1}\right)+\left(q+q_{1}-q\left(\alpha \otimes \alpha^{\prime}\right) q_{1}\right)=0
\end{aligned}
$$

[since $p\left(\alpha \otimes \alpha^{\prime}\right) q_{1}=0$ and $q\left(\alpha \otimes \alpha^{\prime}\right) p_{1}=0$ ]

$$
\text { But } p+p_{1}-p\left(\alpha \otimes \alpha^{\prime}\right) p_{1} \in P \text { and }
$$$$
q+q_{1}-q\left(\alpha \otimes \alpha^{\prime}\right) q_{1} \in Q \text {, and } P \cap Q=\{0\} \text {. }
$$

So, $p+p_{1}-p\left(\alpha \otimes \alpha^{\prime}\right) p_{1}=0$ and

$q+q_{1}-q\left(\alpha \otimes \alpha^{\prime}\right) q_{1}=0$, for any $\alpha \otimes \alpha^{\prime} \in \Gamma \otimes \Gamma$.

Thus $p$ is right quasi regular in $P$ and $q$ is right quasi regular in $Q$, i.e., $p \in J(P)$ and $q \in J(Q)$.

Hence $J\left(V_{1} \otimes V_{2}\right)=J(P) \oplus J(Q)$.

In [4], there is a characterization of Jacobson radical for $\Gamma$-rings in terms of maximal regular left ideals.

\section{Lemma 2.9}

Let $X$ be a $\Gamma$-ring. Then $J(X)=\cap M$, where the intersection is over all maximal regular left ideals $M$ of $X$.

Considering this aspect, we can raise the following problem:

Let the structures of maximal regular left ideals of the operator Banach algebras $R_{1}$ and $L_{2}$ are given. Using this, can we obtain the structure of the Jacobson radical for $V_{1} \otimes_{\gamma} V_{2} ?$

In [6], Behrens radical for $\Gamma$-Banach algebras is introduced which contains the Jacobson radical. Let $\Pi$ denote the class of all subdirectly irreducible $\Gamma$-Banach algebras $V$ such that the intersection of all non-zero ideals of $V$ contains a non-zero idempotent element. The upper radical $R_{B}$ determined by the class $\Pi$ is called the Behrens radical for $V$.

\section{Lemma 2.10}

For a simple $\Gamma$-Banach algebra $V, J(V) \subseteq R_{B}(V)$.

Now, another problem can be raised:

Can we derive analogous result as in Theorem 2.4 in case of the Behrens radical for $V_{1} \otimes_{\gamma} V_{2}$ ?

\section{REFERENCES}

[1] S. Kyuno, "Notes on Jacobson Radicals of Gamma Rings," Mathematica Japonica, Vol. 27, No. 1, 1982, pp. 107-111.

[2] W. E. Coppage and J. Luh, "Radicals of Gamma Rings," Journal of the Mathematical Society of Japan, Vol. 23, No. 1, 1971, pp. 40-52. doi:10.2969/jmsj/02310040

[3] A. C. Paul and A. K. Azad, "Jacobson Radical for Gamma Rings," Rajshahi University Studies Part-B. Journal of Science, Vol. 25, 1977, pp. 153-161.

[4] A. C. Paul and Md. S. Uddin, "On Jacobson Radical for Gamma Rings," Ganit: Journal of Bangladesh Mathematical Society, Vol. 29, 2009, pp. 147-160.

[5] K. N. Raghavan, "The Jacobson Density Theorem and Applications," 2005. http://www.imsc.res.in

[6] H. K. Nath, "A Study of Gamma-Banach Algebras," Ph.D. Thesis, Gauhati University, Guwahati, 2001.

[7] W. E. Barnes, "On the $\Gamma$-Rings of Nobusawa," Pacific Journal of Mathematics, Vol. 18, No. 3, 1966, pp. 411- 
422.

[8] D. K. Bhattacharya and A. K. Maity, "Semilinear Tensor Product of $\Gamma$-Banach Algebras," Ganita, Vol. 40, No. 2, 1989, pp. 75-80.

[9] F. F. Bonsall and J. Duncan, "Complete Normed Algebras," Springer-Verlag, Berlin, 1973. doi:10.1007/978-3-642-65669-9

[10] G. L. Booth, “Operator Rings of a $\Gamma$-Ring," Math Japonica, Vol. 31, No. 2, 1986, pp. 175-183.
[11] N. J. Divinsky, "Rings and Radicals," George Allen and Unwin, London, 1965.

[12] N. Goswami, "Some Results on Operator Banach Algebras of a $\Gamma$-Banach Algebra," Journal of Assam Academy of Mathematics, Vol. 1, 2010, pp. 40-48.

[13] N. Goswami, "On Levitzkinil Radical of Gamma Banach Algebras", Global Journal of Applied Mathematics and Mathematical Sciences, 2012, in Press. 